\title{
Exploratory study of limnological parameters during the cycle of tambaqui fingerlings
}

Estudo exploratório dos parâmetros liminológicos durante o ciclo de cultivo de alevinos de tambaqui

\author{
Fabiano Moreira Figueiredo ${ }^{1}$, Satia Costa Bomfim ${ }^{2}$, Renato Abreu Lima ${ }^{3}$, Wesley Paulo Pontes ${ }^{4}$, Rute

\section{Bianchini Pontuschka ${ }^{5}$ e Fernanda Bay Hurtado ${ }^{6}$}

\author{
1,2,4,5,6 Universidade Federal de Rondônia, RO, Brasil \\ 3 Universidade Federal de Amazonas, AM, Brasil \\ renatoabreu07@hotmail.com
}

\begin{abstract}
As the world population's exponential growth in food security is put at risk, fish farming is one of the economic and effective alternatives in the production of noble and high protein food. For the success the monitoring of the quality of the water during the cycle is fundamental. The present study aimed at the characterization of the limnological parameters in tambaqui nursery (Colosoma macropomum). The study was carried out in the fish farm of Valle del Rio Machado in Presidente Médici - RO, from August/2015 through Junhe/2016, at 08 collection points: water supply and nurseries. The results showed that the parameters such as $\mathrm{pH}$ (6.1 to 8.8), ammonia (0.0 to $\left.1.5 \mathrm{mg} . \mathrm{L}^{-1}\right)$, nitrite $\left(0.0\right.$ to $\left.0.2 \mathrm{mg} \cdot \mathrm{L}^{-1}\right)$, turbidity $(4.1$ to 62.0), were in accordance with what was established by Resolution CONAMA 357/2005; the temperature $\left(28.2\right.$ to $\left.38.2^{\circ} \mathrm{C}\right)$, transparency (18 to $70 \mathrm{~cm}$ ), and alkalinity (10 to $\left.141 \mathrm{mg} \cdot \mathrm{L}^{-1}\right)$ were within the recommended range for fish farming in the specialized literature; dissolved oxygen ranged from 0.8 to $5.2 \mathrm{mg} \cdot \mathrm{L}^{-1}$, but most of the sampled points were below $5 \mathrm{mg} . \mathrm{L}^{-1}$, the electrical conductivity ranging from 51.9 to $178.2 \mu \mathrm{S} . \mathrm{cm}^{-1}$ above that established by Resolution 357/2005/CONAMA. According to the results, the nurseries with cascade supply, receiving the same management are directly affected and there is thus the need for reformulation of the system in fish farming, so that it remains with individual supply and drainage.
\end{abstract}

Keywords: Colossoma macropomum; Fish farming; Nursery; Water quality

\section{Resumo}

Conforme o crescimento exponencial da população mundial a segurança alimentar é colocada em risco, a piscicultura é uma das alternativas econômicas e eficazes na produção de alimento nobre e com alto valor proteico. Para o sucesso o monitoramento da qualidade da água durante o ciclo é fundamental. O presente estudo objetivou a caracterização dos parâmetros limnológicos em viveiros de alevinagem de tambaqui (Colossoma macropomum). O estudo foi realizado na piscicultura Vale do Rio Machado em Presidente Médici - Rondônia, no período de agosto/2015 a junho/2016, em 08 pontos de coletas: abastecimentos, e os viveiros de alevinagem. Os resultados obtidos demonstraram que os parâmetros como $\mathrm{pH}$ (6,1 a 8,8), amônia $\left(0,0\right.$ a 1,5 $\left.\mathrm{mg} \cdot \mathrm{L}^{-1}\right)$, nitrito $\left(0,0\right.$ a $\left.0,2 \mathrm{mg} \cdot \mathrm{L}^{-1}\right)$, turbidez $(4,1$ a 62,0), estiveram de acordo com o estabelecido pela Resolução CONAMA/Brasil 357/2005; a temperatura $\left(28,2\right.$ a 38,2 $\left.{ }^{\circ} \mathrm{C}\right)$, transparência $(18$ a $70 \mathrm{~cm})$, e alcalinidade $\left(10\right.$ a $\left.141 \mathrm{mg} \cdot L^{-1}\right)$, estiveram dentro da faixa recomendada para piscicultura pela literatura especializada; o oxigênio dissolvido variou entre 0,8 a 5,2 mg. $L^{-1}$, porém a maioria dos pontos amostrados estiveram abaixo de $5 \mathrm{mg}$.L-1, a condutividade elétrica que variou entre 51,9 a 178,2 $\mu$ S.cm-1 acima do estabelecido pela Resolução CONAMA 357/2005. De acordo com os resultados, os viveiros com abastecimento em cascata, recebendo o mesmo manejo são diretamente afetados e existe assim a necessidade de reformulação do sistema na piscicultura, de modo que este fique com abastecimento e escoamento individual. 


\section{Introduction}

Due to the world population growth, the shortage of animal protein sources has become a global concern (PAGGI, 2006), according to the FAO (2016), for the year 2050, it is estimated that the world population 9 billion people, and to feed this growing number of people, the annual production of meat is expected to increase by more than 200 million reach 470 million (SCHULTER; VIEIRA FILHO, 2017). Thus, providing quality food is becoming increasingly difficult, given that population growth is accompanied by high waste generation, which is the major source of environmental degradation (GUERREIRO, 2012).

The biannual publication The State of the World Fisheries and Aquaculture (FAO, 2016) shows the growth of aquaculture, which already represents 73,8 million tons of fish in the world, or about $44 \%$ of world fish production. In Brazil, fish farming is becoming the most effective and economical alternative to produce foods with high nutritional value, generate employment and income and, at the same time, harness natural resources, representing great potential for growth in Brazil (PAGGI, 2006). since this country has adequate attributes for this activity, such as the abundance of water resources; large territory; three-quarters of its geographical area in the tropical zone of Ecuador; abundant solar energy; large number of native species easy to grow, highly profitable for investors (MAR; OLIVEIRA; COSTA, 2012).

Fish farming is becoming the most effective and economic alternative to produce food with high nutritional value, has generated jobs and income, and, at the same time takes advantage of natural resources, representing a great potential for growth in Brazil (PAGGI, 2006), since this country has adequate attributes for this agricultural activity, such as abundant water resources; large territory; three quarters of its geographical area in the tropical zone of the Equator; abundant solar energy; high number of native species that are easy to raise, all of which is highly profitable for investors (MAR; OLIVEIRA; COSTA, 2012).

If this hydrological potential is used correctly, Brazil will soon be among the largest producers of fish, enabling the development of fish processing industries and generating thousands of jobs, thus contributing to food security (GUERREIRO, 2012).

Fish farming had its beginning in the state of Rondônia around the year 1985 with the implementation of this activity in the municipality of Pimenta Bueno, with fingerling distribution to 100 fish farmers (MDIC, 2007).

According to Xavier (2013), the regional climate, the location close to a large consumer market, and the high availability of water that ensure the existing river basins, place the state in the spotlight in Brazilian aquaculture production. According to IBGE (2016), the state of Rondônia ranks first in the ranks of the Federation Units, with 90,636 tons of fish being harvested, with tambaqui (Colossoma macropomum) being the main species produced in captivity.

However, in fish farming, water availability and quality are essential factors, since fish use water to obtain oxygen and to release carbon dioxide, nitrogenous wastes and other substances via excretion. Therefore, the monitoring of water quality in production systems is important for the development of fish (MORO et al., 2013).

Abiotic factors including soil content, sunlight, temperature and atmospheric air are directly related to the aquatic environment. This dynamic process causes variations in the limnological characteristics of fish farms. These variations occur cyclically throughout the year, according to successions and climate changes and also because of aquaculture management (PEREIRA et al., 2012). Thus, this study aimed at characterizing the limnological parameters of water in ponds used for tambaqui (Colossoma macropomum) rearing.

\section{Material and Methods}

\subsection{Study Area}

The research was carried out at Vale do Rio Machado farm, in the rural area of the municipality of Presidente Médici - Rondônia, located at the geographic coordinates $11^{\circ} 03^{\prime} 43.5^{\prime \prime} \mathrm{S}$ and $61^{\circ} 53^{\prime} 54.4^{\prime \prime} \mathrm{W}$, which is used for fish farming. The lagoons are supplied with water from a source belonging to the property, 
located upstream of the fish farm, passing through two water supply reservoirs, which are later distributed to the lagoons, whose depths vary from 1.50 to 1.90 meters.

This study was conducted between August 2015 and June 2016, with monthly collections performed in the mornings. A total of eight collection points (supply reservoirs 01 and 02 ; and ponds $01,02,03,04,12$ and 13) were assessed (Figure 1).

Figure 1 - Vale do Rio Machado fish farm

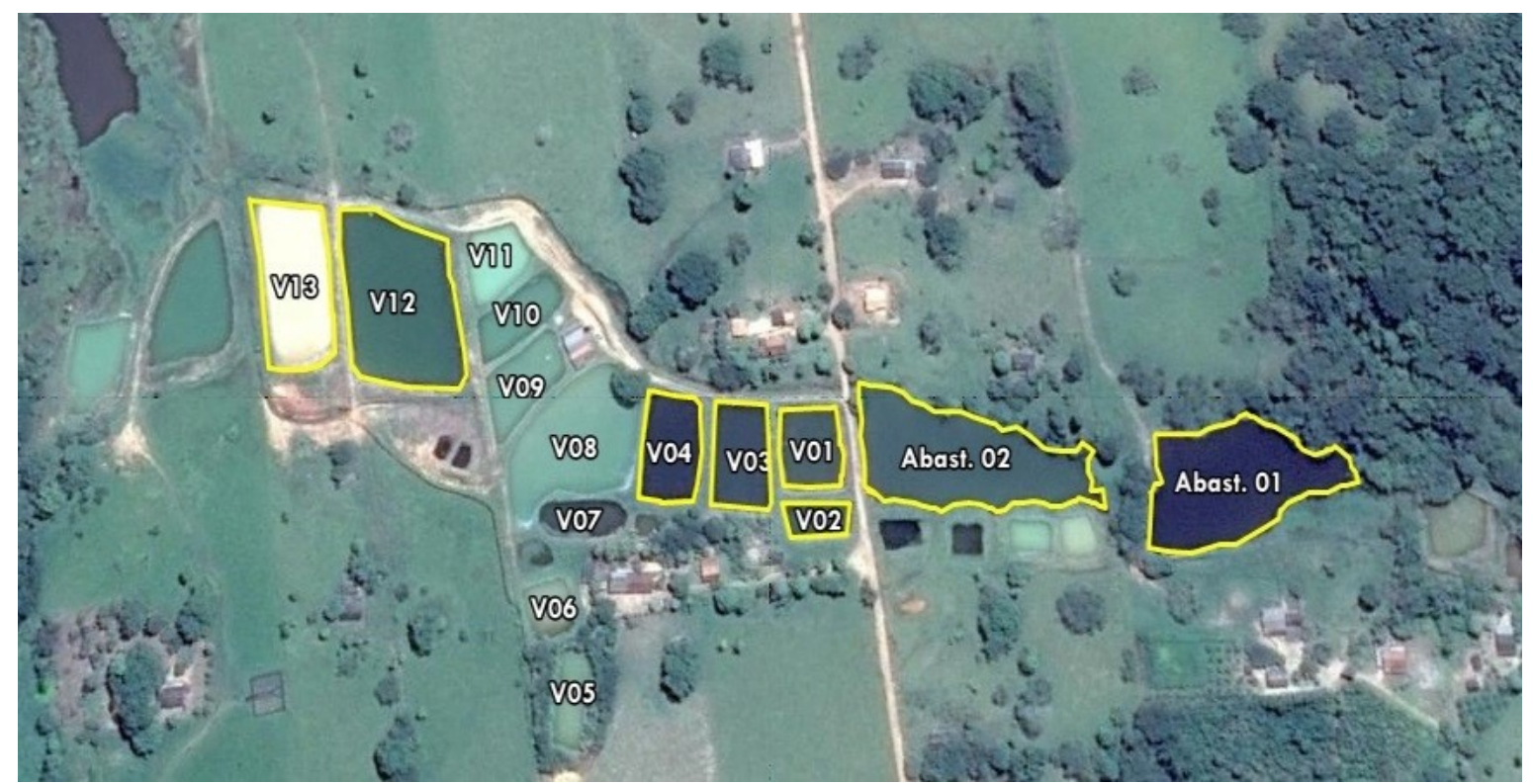

( $\mathrm{V}=$ Ponds; Abast. $=$ Supply reservoirs)

Source: Adapted from Google Earth (accessed on June 3/2016)

To select the sampling points, we took into account the nursery supply, which is interconnected "cascade type". In this context, sampling occurred in the center of each nursery $30 \mathrm{~cm}$ from the surface, except in the supply dams, where it was shown only near the exit point, for each sampling point three samples were collected in the volume of $1.0 \mathrm{~L}$. selection of the samples in each nursery was based on the literature of Bicudo (2004), which states that in shallow environments (about 2 meters) are sampled at the surface and at the bottom or only at the surface, assuming no vertical structuring.

The cycle of fingerlings production is constant in the pisciculture because the method of spawning by hormonal induction is used. In this way there are fingerlings for commercialization all year in this fish culture.

\subsection{Analysis of limnological parameters}

Temperature $\left({ }^{\circ} \mathrm{C}\right)$ and dissolved oxygen levels $\left(\mathrm{mg} . \mathrm{L}^{-1}\right)$ were analyzed using an oxygen meter (LT lutron DO-5519) and transparency (cm) was assessed using secchi disks, both of which were measured in loco, whereas the following parameters were assessed at the Laboratory of Physicochemical and Microbiological Analyses (LAFQM), Presidente Médici Campus: $\mathrm{pH}$, measured by a $\mathrm{pH}$ meter (mPA-210); conductivity ( $\left.\mu \mathrm{S} . \mathrm{L}^{-1}\right)$ measured using a conductivimeter (LUCA-150); turbidity (UNT), measured using a turbidimeter (HOMIS 953); and ammonia (mg.L-1 of NH3), alkalinity (mg.L.- of $\mathrm{CaCO}_{3}$ ) and nitrite levels (mg.L ${ }^{-1}$ of $\mathrm{NO}_{2}{ }^{-1}$ ), which were assessed through a photometer (Lovibond MULTIDIRECT). A specific kit was used for each parameter.

\subsection{Statistical analysis}

The mean values were analyzed using the software Graph Prism Instat $3{ }^{\circledR}$ through analysis of variance (ANOVA) and by the Tukey post-test. $P$ values $<0.05$ were considered indicative of significance. 


\section{Results and discussion}

\subsection{Physicochemical parameters}

The values of dissolved oxygen, temperature, alkalinity, nitrite, ammonia and electrical conductivity, obtained by analysis of variance (ANOVA) presented significant differences between the evaluated months (Table 1 and Figure 2).

When compared among different ponds, the following parameters exhibited significant differences: $\mathrm{pH}$, dissolved oxygen, transparency, alkalinity, nitrite, ammonia, and electrical conductivity, obtained by analysis of variance (ANOVA), while temperature and turbidity values did not present significant differences among the ponds (Table 2 and Figure 3).

The monthly variations of these parameters are related to precipitation and temperature peaks during the study period. The limnological variations at each collection point may be related to the depth of the ponds and wind action, causing small displacements in the layers of water, hence mixing the water at the surface with the water at the bottom of the pond (CAMPECHE et al., 2009).

Figure 2 - Limnological parameters of water per month of the nurseries sampled

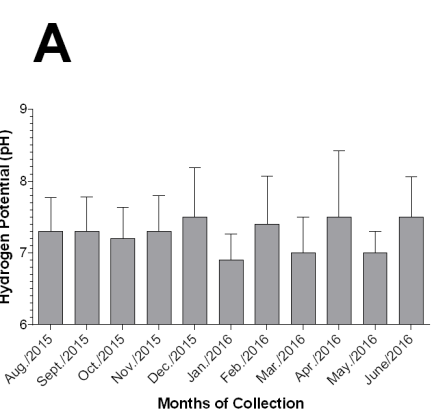

\section{B}

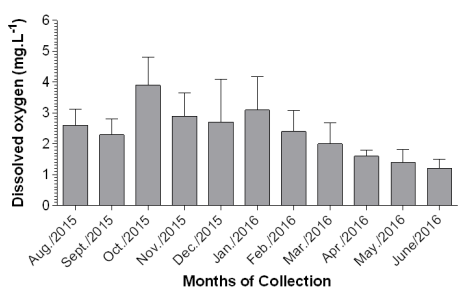

E
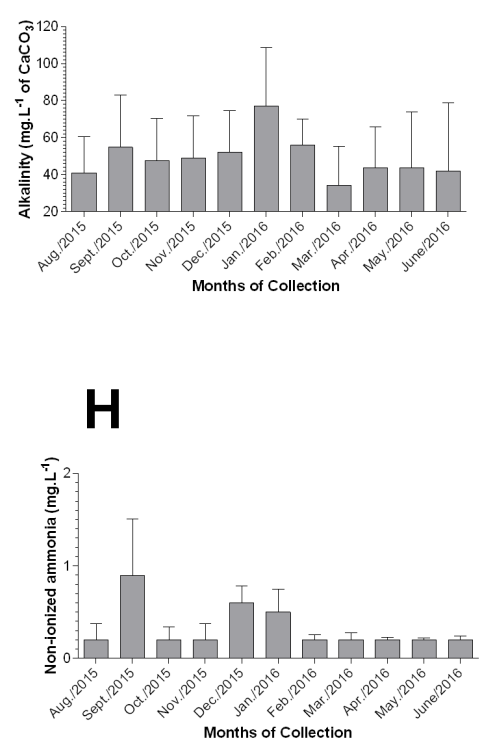

C

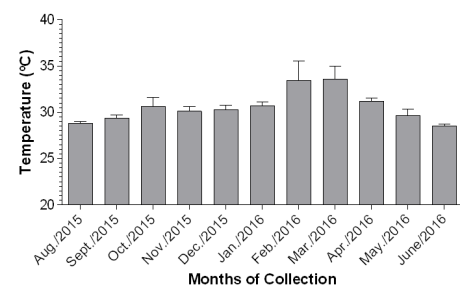

$\mathbf{F}$
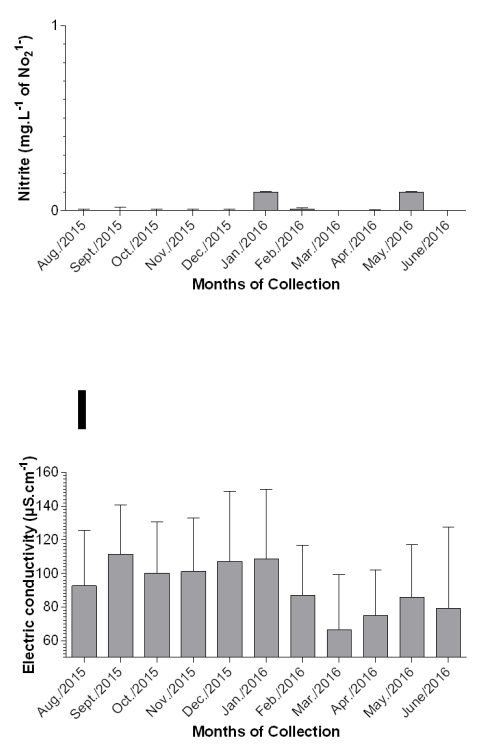

Where: A) Hydrogenionic potential; B) Dissolved oxygen (mg. $\mathrm{L}^{-1}$ ); C) Water temperature $\left.\left({ }^{\circ} \mathrm{C}\right) ; \mathrm{D}\right)$ Transparency (cm); E) Alkalinity (mg. $\mathrm{L}^{-1}$ of $\left.\mathrm{CaCO}_{3}\right)$; F) Nitrite (mg.L-1); G) Turbidty (NTU); H) Non-ionized ammonia (mg.L $\left.\left.\mathrm{L}^{-1}\right) ; \mathrm{I}\right)$ Electrical conductivity $\left(\mu \mathrm{S} . \mathrm{cm}^{-1}\right)$

Source: Research data, 2016 


\begin{tabular}{|c|c|c|c|c|c|c|c|c|c|c|}
\hline 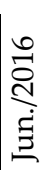 & 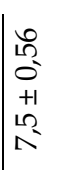 & 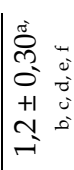 & 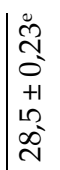 & 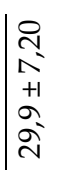 & $\mid \begin{array}{l}0 \\
0 \\
0 \\
0 \\
0 \\
+1 \\
0 \\
0 \\
7 \\
7\end{array}$ & $\mid \begin{array}{l}1 \\
8 \\
0 \\
0 \\
+1 \\
0 \\
0\end{array}$ & $\mid \begin{array}{l}8 \\
\infty \\
+1 \\
+1 \\
6 \\
0 \\
0\end{array}$ & 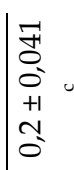 & 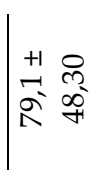 & II \\
\hline 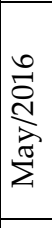 & $\begin{array}{l}0 \\
0 \\
0 \\
+1 \\
0 \\
1 \\
1\end{array}$ & 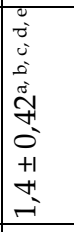 & $\begin{array}{l}\overrightarrow{0} \\
\hat{0} \\
0 \\
+1 \\
0 \\
0 \\
2 \\
i\end{array}$ & $\begin{array}{l}\infty \\
0 \\
\infty \\
+1 \\
0 \\
\infty \\
\infty \\
\infty\end{array}$ & 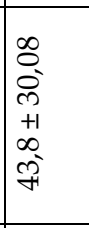 & $\begin{array}{c}0 \\
0 \\
0 \\
+1 \\
-1 \\
0\end{array}$ & 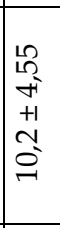 & $\begin{array}{l}0 \\
0 \\
0 \\
0 \\
+1 \\
0 \\
0 \\
0\end{array}$ & $\begin{array}{l}\infty \\
n \\
\infty \\
+1 \\
+\infty \\
\infty \\
\infty\end{array}$ & 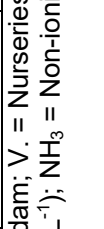 \\
\hline 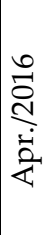 & 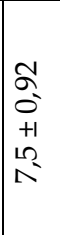 & 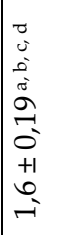 & 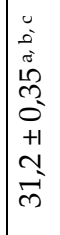 & $\begin{array}{l}10 \\
\curvearrowright \\
\sigma \\
+1 \\
\infty \\
\infty \\
\infty\end{array}$ & 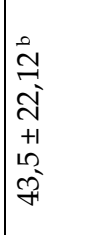 & $\begin{array}{c}1 \\
\delta \\
0 \\
+1 \\
0 \\
0 \\
0\end{array}$ & $\begin{array}{l}0 \\
10 \\
\infty \\
0 \\
+1 \\
0 \\
10 \\
1\end{array}$ & 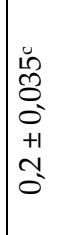 & 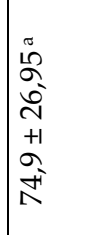 & 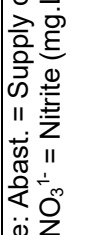 \\
\hline 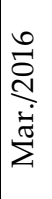 & $\begin{array}{l}0 \\
0 \\
0 \\
0 \\
+1 \\
0 \\
1 \\
\end{array}$ & $\begin{array}{l}\hat{0} \\
0 \\
+1 \\
0 \\
\hat{i}\end{array}$ & \begin{tabular}{l}
0 \\
\multirow{+}{*}{} \\
-1 \\
+1 \\
+1 \\
0 \\
0 \\
0
\end{tabular} & $\begin{array}{l}\infty \\
2 \\
\infty \\
+1 \\
\hat{a} \\
\text { ते }\end{array}$ & $\begin{array}{l}0 \\
\infty \\
0 \\
\vec{N} \\
+1 \\
+1 \\
0 \\
0 \\
\infty \\
0\end{array}$ & $\begin{array}{c}0 \\
0 \\
0 \\
+1 \\
0 \\
0\end{array}$ & 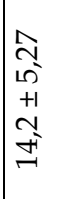 & $\begin{array}{l}0 \\
0 \\
0 \\
0 \\
0 \\
+1 \\
1 \\
0 \\
0\end{array}$ & $\begin{array}{l}0 \\
\sigma \\
\alpha \\
\tilde{\omega} \\
+1 \\
0 \\
0 \\
0 \\
0\end{array}$ & 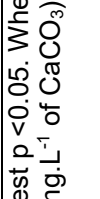 \\
\hline 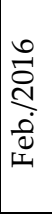 & \begin{tabular}{l}
2 \\
2 \\
0 \\
+1 \\
+1 \\
\multirow{1}{*}{}
\end{tabular} & 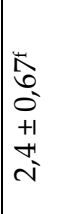 & 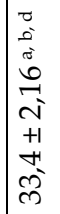 & $\begin{array}{l}\text { I } \\
\infty \\
\infty \\
+1 \\
0 \\
\infty \\
\infty \\
\infty\end{array}$ & \begin{tabular}{l}
$\hat{N}$ \\
\multirow{+}{*}{} \\
+1 \\
$\infty$ \\
$\infty$ \\
10 \\
10
\end{tabular} & $\begin{array}{l}20 \\
0 \\
0 \\
0 \\
+1 \\
0 \\
0 \\
0\end{array}$ & 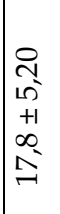 & $\begin{array}{l}0 \\
0 \\
0 \\
0 \\
+1 \\
1 \\
0 \\
0\end{array}$ & 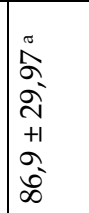 & 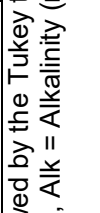 \\
\hline 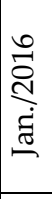 & $\begin{array}{l}0 \\
\infty \\
0 \\
+1 \\
+1 \\
\alpha \\
0\end{array}$ & 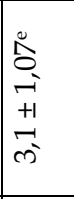 & $\begin{array}{l}0 \\
\tilde{\sigma} \\
\tilde{\sigma} \\
0 \\
+1 \\
+1 \\
\hat{0} \\
0 \\
0\end{array}$ & $\begin{array}{l}\infty \\
\infty \\
\infty \\
+1 \\
\infty \\
\infty \\
\infty\end{array}$ & 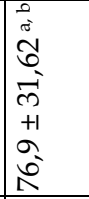 & 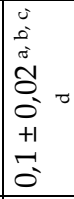 & 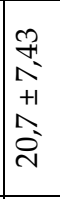 & $\begin{array}{l}\frac{1}{9} \\
0 \\
0 \\
+1 \\
0 \\
0 \\
0\end{array}$ & 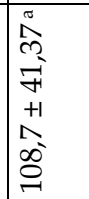 & 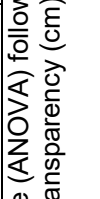 \\
\hline 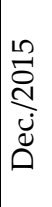 & 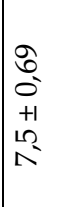 & 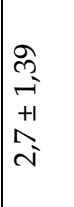 & $\begin{array}{l}\text { Oे } \\
0 \\
+1 \\
0 \\
0 \\
0 \\
0\end{array}$ & 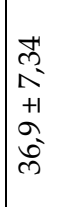 & 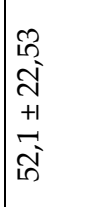 & $\begin{array}{l}2 \\
\delta \\
0 \\
+1 \\
0 \\
0 \\
0\end{array}$ & $\begin{array}{l}\sqrt{1} \\
\infty \\
+ \\
+1 \\
0 \\
0 \\
0 \\
-1\end{array}$ & $\begin{array}{l}0 \\
0 \\
0 \\
0 \\
0 \\
+1 \\
0 \\
0 \\
0\end{array}$ & 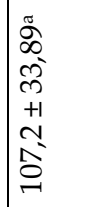 & 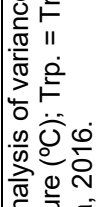 \\
\hline 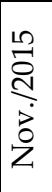 & $\begin{array}{l}0 \\
10 \\
0 \\
+1 \\
+1 \\
2 \\
12\end{array}$ & $\begin{array}{l}\tilde{v} \\
0 \\
0 \\
0 \\
+1 \\
a \\
\hat{N}\end{array}$ & 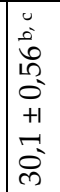 & $\begin{array}{l}8 \\
0 \\
6 \\
+1 \\
\vdots \\
0 \\
0\end{array}$ & 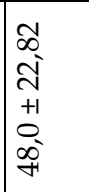 & $\begin{array}{l}\pi \\
\tilde{\sigma} \\
0 \\
0 \\
+1 \\
0 \\
0 \\
0\end{array}$ & $\begin{array}{l}\infty \\
N \\
\infty \\
+1 \\
+1 \\
= \\
= \\
=\end{array}$ & $\begin{array}{l}\infty \\
0 \\
0 \\
0 \\
+1 \\
+1 \\
0 \\
0\end{array}$ & 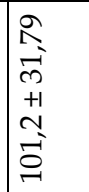 & 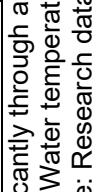 \\
\hline 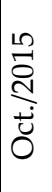 & 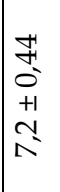 & 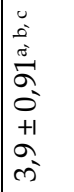 & $\begin{array}{l}0 \\
\hat{\alpha} \\
0 \\
+1 \\
+1 \\
0 \\
0 \\
0 \\
\text { ల. }\end{array}$ & 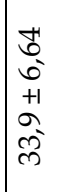 & 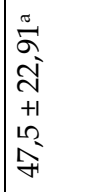 & $\begin{array}{l}\check{a} \\
\delta \\
0 \\
+1 \\
+1 \\
0 \\
0\end{array}$ & 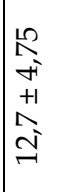 & $\begin{array}{l}\not \\
0 \\
0 \\
0 \\
+1 \\
\vdots \\
0 \\
0\end{array}$ & $\begin{array}{l}8 \\
0 \\
0 \\
0 \\
+1 \\
+1 \\
0 \\
8 \\
\end{array}$ & 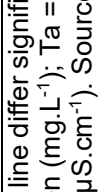 \\
\hline 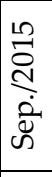 & $\begin{array}{l}\infty \\
0 \\
0 \\
0 \\
+1 \\
0 \\
2 \\
1 \\
1\end{array}$ & 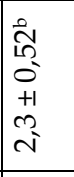 & 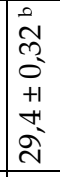 & $\begin{array}{l}\qquad 0 \\
\sigma \\
\sigma \\
+1 \\
0 \\
\text { के }\end{array}$ & $\begin{array}{l}0 \\
N \\
\infty \\
0 \\
+1 \\
+1 \\
\infty \\
\mathbb{1} \\
10\end{array}$ & $\begin{array}{l}\stackrel{2}{0} \\
0 \\
0 \\
+1 \\
0 \\
0 \\
0\end{array}$ & $\begin{array}{l}\hat{\alpha} \\
\alpha \\
+1 \\
+1 \\
+1 \\
\sigma \\
-1\end{array}$ & $\begin{array}{l}0 \\
0 \\
0 \\
0 \\
0 \\
0 \\
+1 \\
0 \\
0 \\
0\end{array}$ & 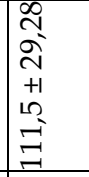 & : \\
\hline 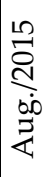 & $\begin{array}{l}\text { 今 } \\
0 \\
+1 \\
\text { Oे } \\
\end{array}$ & $\begin{array}{l}\tilde{0} \\
0 \\
0 \\
0 \\
+1 \\
0 \\
0 \\
i\end{array}$ & 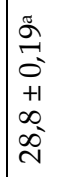 & 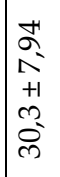 & 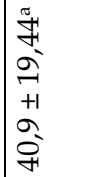 & $\begin{array}{l}\tilde{\sigma} \\
0 \\
0 \\
0 \\
+1 \\
0 \\
0 \\
0\end{array}$ & $\begin{array}{l}\hat{\alpha} \\
\hat{\infty} \\
+1 \\
0 \\
0 \\
0 \\
0\end{array}$ & $\begin{array}{l}\tilde{a} \\
\infty \\
0 \\
0 \\
0 \\
+1 \\
2 \\
0 \\
0\end{array}$ & 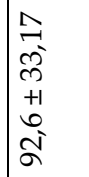 & 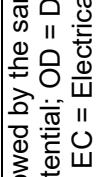 \\
\hline 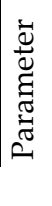 & $\frac{I_{2}}{2}$ & ค & $\stackrel{\sigma}{F}$ & $\dot{\vec{\theta}}$ & $\ddot{\ddot{z}}$ & Õ & 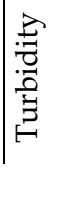 & 愛 & U. & 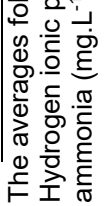 \\
\hline
\end{tabular}




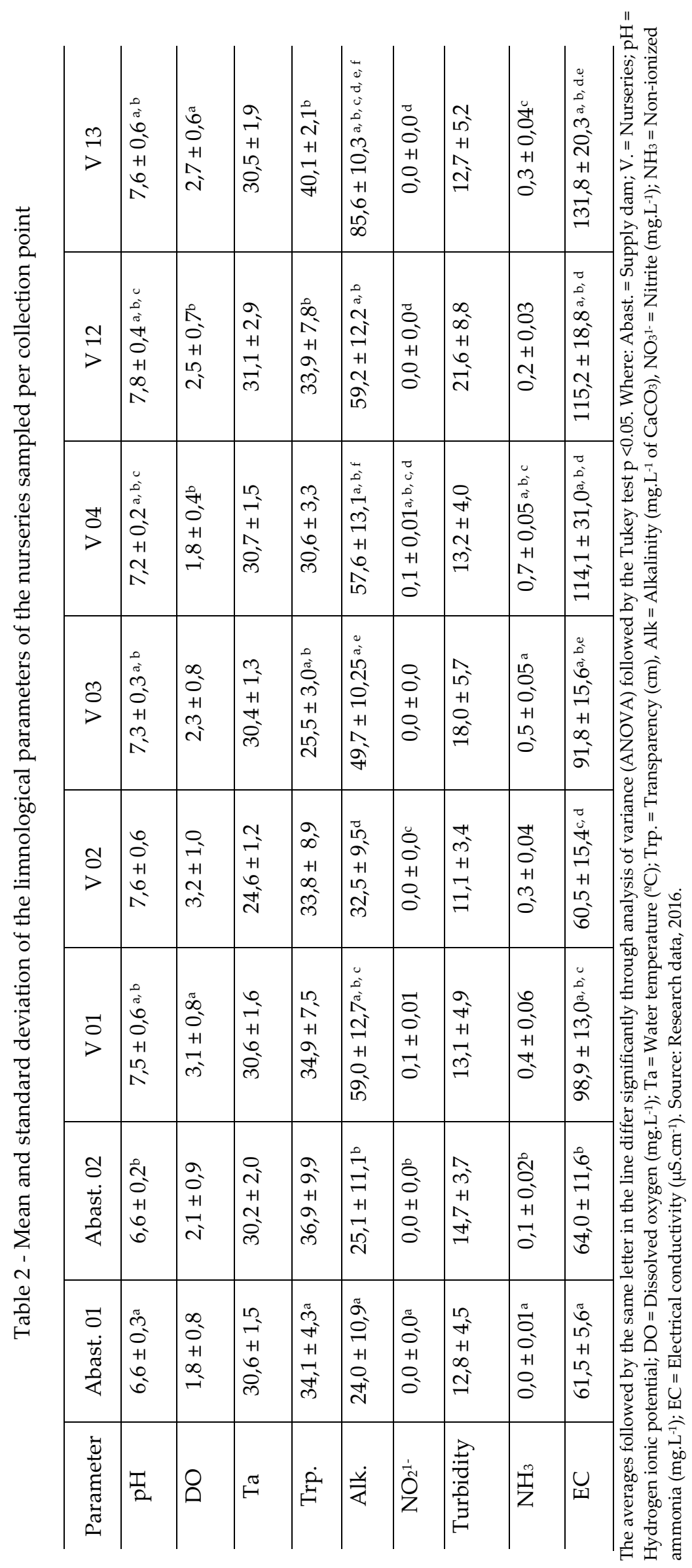


Figure 3 - Variation of the liminological parameters by point of collection

A

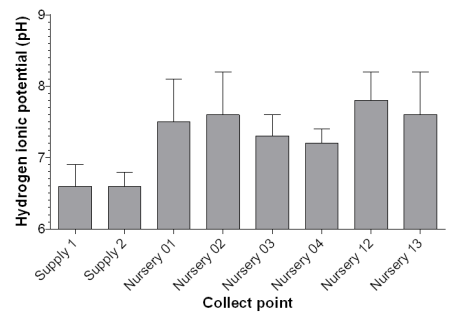

D

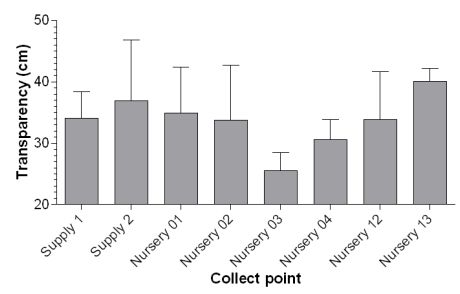

G

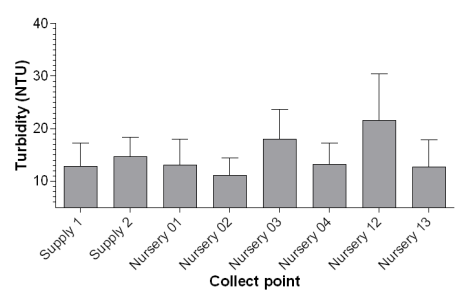

B

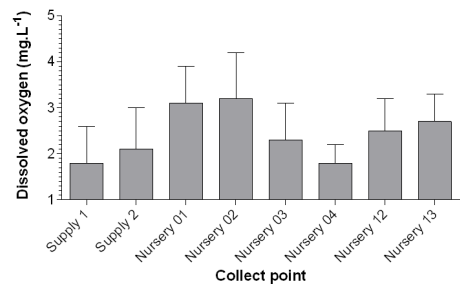

$\mathbf{E}$

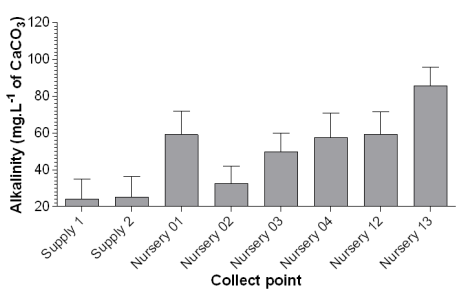

H

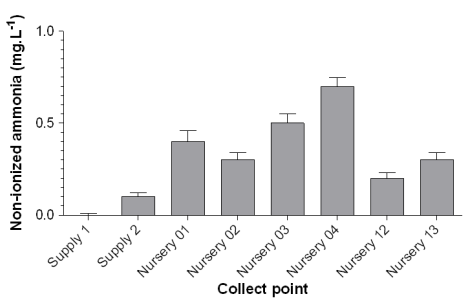

C

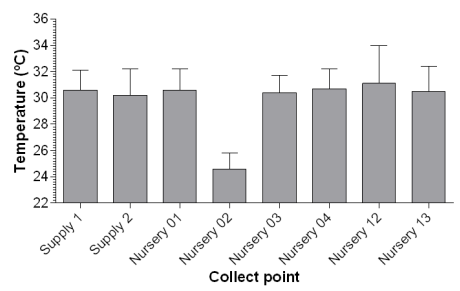

$\mathbf{F}$
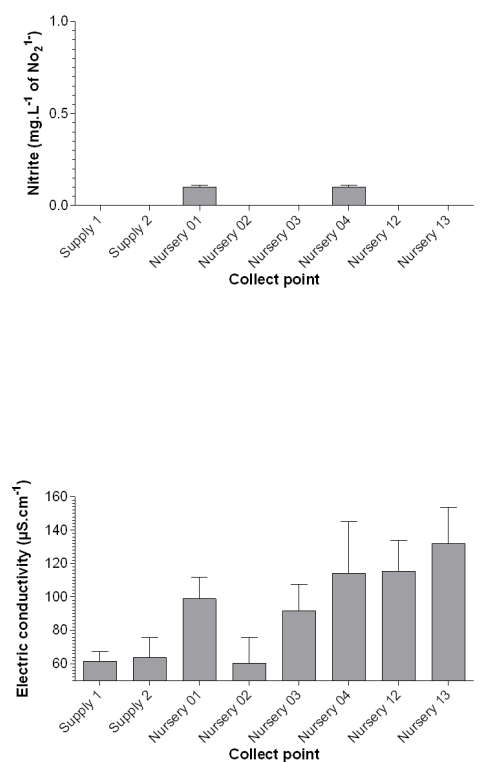

Where: A) Hydrogenionic potential; B) Dissolved oxygen (mg. $\left.\mathrm{L}^{-1}\right)$; C) Water temperature $\left({ }^{\circ} \mathrm{C}\right)$; D) Transparency (cm); E) Alkalinity (mg.L-1 of $\left.\left.\mathrm{CaCO}_{3}\right) ; \mathrm{F}\right)$ Nitrite (mg.L-1) ; G) Turbidty (NTU); H) Non-ionized ammonia (mg. $\left.\left.\mathrm{L}^{-1}\right) ; \mathrm{I}\right)$ Electrical conductivity $\left(\mu \mathrm{S} . \mathrm{cm}^{-1}\right)$

Source: Research data, 2016

\subsection{Potential of hydrogen $(\mathrm{pH})$}

The $\mathrm{pH}$ varied from slightly acidic to alkaline (6.1 to 8.8). Thus, there were no significant differences among the averages of the samples from the water supply reservoirs and the ponds during the evaluated months (Table 1 and Figure 2). Nevertheless, no significant differences were observed between V02 and the other spots. However, the rest of the spots presented significant differences among them, as shown in Table 2 and Figure 3.

The minimum and maximum values were recorded in April 2016, wherein the minimum was recorded in supply reservoir 02 , with the lowest $\mathrm{pH}$ values during the study, whereas the maximal value was recorded in pond 12, so that the $\mathrm{pH}$ upstream of the fish farm, which is slightly acidic, became more alkaline when it entered the fish farm. This $\mathrm{pH}$ change might be related to the producer's management (pond liming and fertilization). 
The $\mathrm{pH}$ values similar to these were also recorded in other investigations in the state of Rondônia, such as in the study by Costa (2014), with limnological parameters assessed at a fish farm located in the municipality of Alvorada D'Oeste, which obtained $\mathrm{pH}$ values between 6.4 and 9.4. The levels found by Paiva (2014), in a case study in the municipality of Ji-Paraná, with pH values between 5.69 and 7.32 also agree with those from the present study. Hurtado et al., (2018) also observed similar values (6.4 to 9.1) in an investigation conducted in the Tancredópolis district, where tambaqui fish were cultured with a continuous water flow supply.

The $\mathrm{pH}$ values in the study carried out at the Vale do Rio Machado fish farm comply with the range recommended by Kubtiza (2003), with $\mathrm{pH}$ values between 6.5 and 8.0, i.e., near neutrality, since $\mathrm{pH}$ values below or above this range may be detrimental to overall fish health conditions and growth.

These $\mathrm{pH}$ values obtained are also in agreement with the reference values (between 6.0 and 9.0) proposed by Resolution 357/2005/CONAMA. According to Ceccarelli, Senhorini and Volpato (2000), tambaqui is a tropical fish, and the $\mathrm{pH}$, therefore, must range between 7.0 and 8.0, which are values similar to those specific for aquaculture cited by Sipaúba-Tavares (1994), who stated that pH values between 5.0 and 6.0 cause delayed growth; between 7.0 and 8.5, adequate growth; and $\mathrm{pH}$ values > 10, delayed growth.

Parron, Muniz and Pereira (2011) emphasize that the $\mathrm{pH}$ is the most important limnological parameter, as well as the most frequently used element in water quality analysis since this factor directly influences the aquatic ecosystems because of its effects on fish physiology.

\subsection{Dissolved oxygen (DO)}

The DO varied between 0.8 and $5.2 \mathrm{mg} . \mathrm{L}^{-1}$, with the maximum in pond 13 in October 2015 and the minimum in pond 13 in May 2016 (Table 1 and Figure 2). Among the means of the evaluated months there were no significant differences between December 2015 and March 2016 in relation to the reamining months (Table 1); however, in relation to the sampling sites there was a significant difference among ponds V01, V13, V04 and V12 (Table 2 and Figure 3). The rest of the sampling spots did not present significant differences.

It may be suggested that the months with the highest temperatures correlated with lower DO values, since DO solubility decreases in the water as temperature rises (AYROZA, 2012).

The values obtained for this parameter agree with studies carried out in neighboring regions, such as the investigation by Costa (2014), with variations of DO between 0.95 and 7.5 mg.L-1; Paiva (2014) with

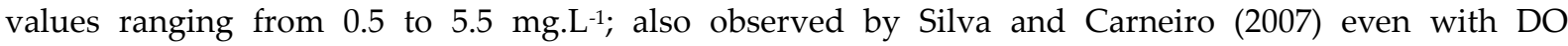
concentrations considered low, ranging from 0.2 to $6.5 \mathrm{mg} . \mathrm{L}^{-1}$; Hurtado et al., (2018), who studied the rearing of tambaqui in sequentially laid out ponds, obtained results ranging from 2.5 to $5.1 \mathrm{mg}^{-\mathrm{L}^{-1}}$; Silva et al. (2017) who studied the polyculture of species in ponds also obtained averages between 3.15 and 5.84 mg.L-1; none of the above-mentioned studies reported mortality.

For DO Resolution 357/2005/CONAMA establishes a minimum value of 5 mg.L-1; based on this value, and comparing it with the results obtained in the present study, we observed that, except for in pond 01 during December 2015, and in pond 13 during October 2015, the remaining values analyzed were below the value established by the resolution cited.

Low DO concentrations are known to cause morphological changes in fish, of which dermal prolongation in the inferior jaw is one of the main modifications caused in tambaqui fish. This modification is observed within a few hours (2 to 3 hours) increasing the fish's ability to harness DO in the water-air interface (ESTEVES; FURTADO, 2011).

\subsection{Temperature ( $\mathrm{T})$}

Since each fish species expresses different physiological characteristics, each of them are adapted to a different temperature, which is called their optimal temperature. Because tambaqui is a tropical species, it presents an optimal temperature between 27 and $32{ }^{\circ} \mathrm{C}$, as stated by Boyd (1997).

Water temperature during the study period varied between 28.2 and $38.2{ }^{\circ} \mathrm{C}$ with a maximum temperature in V12, during February $2016\left(38.2^{\circ} \mathrm{C}\right)$ and the minimum in V13, during June 2016. Significant differences were only observed in December 2015 in relation to the remaining months (Table 1 and Figure

2); however, no significant differences among the sampling sites were observed (Table 2 and Figure 3). Silva 
and Fujimoto (2015) obtained temperatures varying from 25.5 to $32.2{ }^{\circ} \mathrm{C}$ for water temperatures of a tambaqui culture in fishponds, in the city of Aracaju; Hurtado et al. (2018) reported temperatures between 27.8 and $29.1{ }^{\circ} \mathrm{C}$ for tambaqui cultures in excavated tanks.

Because tambaqui is a tropical species, it easily adapts to culture systems in regions with high temperatures. Studies carried out by Nakatani et al.n (2001) and Piana, Baumgartner and Gomes (2003) showed that rearing temperatures between 26 and $30^{\circ} \mathrm{C}$ resulted in a higher total length and weight increase for tropical and subtropical species.

Baldisserotto (2013) states that the limits of thermal tolerance in fish are not fixed values, because, when exposed to temperatures close to those considered lethal, they acquire a certain degree of adaptation. The author also states that thanks to this adaptation, the temperature that would have previously killed the fish is tolerated by it, a process called acclimatization.

\subsection{Transparency (CM)}

The transparency varied between 18 and $70 \mathrm{~cm}$, with the highest values recorded in December 2015, in V13 $(70 \mathrm{~cm})$, which consequently occurred in months with high rainfall indexes, resulting in a faster and more constant water renewal in the tanks. The minimum value occurred in V01, during June $2016(18 \mathrm{~cm})$, the month presenting the lowest precipitation.

There were no significant differences among the evaluated months (Table 1 and Figure 2); however, regarding the sampling points, significant differences were observed in supply reservoirs 1 and V03; V12 and V13 (Table 2 and Figure 3), which are related to the management practices employed by the owners in each pond.

Transparency values between 40 and $80 \mathrm{~cm}$ are considered adequate for nursing tambaqui and other omnivorous species. This parameter is paramount during the nursing phase, as it enables the quantification of phytoplankton and zooplankton, which are used as natural foods and are highly important to the fingerlings' nutrition.

Silva (2014), who carried out a limnological study in the Carlos Eduardo Matize dam in Presidente Médici, state of Rondônia between August 2013 and June 2014, also observed similar results in relation to transparency, which peaked in January, March and May, months characterized by high rainfall in the region during the study period.

\subsection{Alkalinity $\left(\mathrm{CACO}_{3}{ }^{2-}\right)$}

Among the evaluated months, there were no significant differences in September 2015, November 2015, December 2015, February 2015, February 2016 and May 2016 (Table 1 and Figure 2). However, with relation to the sampling sites, significant differences were recorded between supply reservoir $01, \mathrm{~V} 01$ and V03; supply reservoir 02, V01, V04, V12 and V13; V01 and V13; V02 and V13; V3 and V13; V04 and V13 (Table 2 and Figure 3). Thus, observed alkalinity levels presented significant differences among the sampling sites owing to different management practices employed in each pond after the end of the nursing period by the fish farmer.

The low levels presented in supply reservoir 01 may be related to the fact that the dam is used to supply the system, and that no chemical or organic treatment occurred during the study period. The high alkalinity levels observed in the last pond are correlated with the discharge from other ponds owing to the sequential flow system, characterized by water flowing from one tank to another without prior treatment (PAGGI, 2006).

Even with varying alkalinity levels, these values agree with those indicated by Souza (2014) and Queiroz and Silveira (2006), between 20 and $300 \mathrm{mg} . \mathrm{L}^{-1}$. These levels are also similar to those found by Costa (2014), who carried out limnological analyses in a fish farm used for raising tambaqui, with alkalinity levels between 50 and $195 \mathrm{mg}$ of $\mathrm{CaCO}_{3} \cdot \mathrm{L}^{-1}$.

\subsection{Nitrite $\left(\mathrm{NO}_{2}{ }^{1-}\right)$}

Nitrite is an intermediary compound in the nitrification process, in which ammonia is oxidated by bacteria, resulting in the formation of nitrite and subsequently, nitrate $\left(\mathrm{NO}_{3}{ }^{1-}\right)$. When absorbed by fish, 
nitrite causes the oxidation of blood hemoglobin, which turns into methemoglobin. This molecule, unlike hemoglobin, is not able to transport oxygen, and for this reason, excess nitrite in the water causes severe physiological and respiratory problems (MORO et al., 2013).

Nitrite levels varied between 0.0 and $0.2 \mathrm{mg} . \mathrm{L}^{-1}$. The averages of the analyzed months showed no significant differences among December 2015, February 2016, March 2016, April 2016, May 2016 and June 2016 in relation to the remaining months (Table 1 and Figure 2). However, with relation to the sampling sites, there were significant differences between supply reservoir 01 and V04; supply reservoir 02 and V04; V02 and V04; V04 and V12 and V13 (Table 2 and Figure 3). The rest of the sampling sites showed no significant differences.

These nitrite levels are similar to those observed by Souza (2007) in a case study on tilapia rearing, and those obtained by Campeche et al., (2009), with values ranging from $0.023 \mathrm{mg} . \mathrm{L}^{-1}$ to $0.178 \mathrm{mg} . \mathrm{L}^{-1}$ and $0.066 \mathrm{mg} . \mathrm{L}^{-1}$ to $0.386 \mathrm{mg} . \mathrm{L}^{-1}$, respectively. Cavero et al. (2004), who assessed the increase in ammonia concentration in pirarucu juveniles reared in a confined environment, found values between $2.3 \times 10^{-4}$ and $3 \times 10^{-4} \mathrm{mg} . \mathrm{L}^{-1}$, highlighting that such values are not considered toxic enough to affect the physiological requirements of fish.

The maximum nitrite level tolerated by most species is $0.5 \mathrm{mg} \cdot \mathrm{L}^{-1}$ and values above this range are known to cause fish mortality. Overall, high nitrite levels in the water are caused by inadequate feeding management, in which excess food is provided and not totally consumed, along with excess fertilization in some cases (MORO et al., 2013).

According to Resolution 357/2005/CONAMA, nitrite levels must not exceed $1 \mathrm{mg} . \mathrm{L}^{-1}$. Therefore, the levels found in this study comply with fish farm standards and thus do not impair the growth and immunoresistance of fish, as claimed by Kubitza (2003), who stated that high nitrite concentrations (0.3 to $0.5 \mathrm{mg} . \mathrm{L}^{-1}$ ) can cause a decrease in fish growth and in resistance to diseases.

\subsection{Turbidity (UNT)}

Turbidity levels varied between 4.1 and $62.0 \mathrm{UNT}$, and no significant differences were observed among the collection month means (Table 1 and Figure 2). In relation to the sampling sites, significant differences were not observed either (Table 2 and Figure 3), complying with the standards established by Resolution 357/2005/CONAMA (100 UNT).

Such high turbidity values in the water supply reservoirs are related to the fact that the water source that supplies the fish farm is located in a landform which favors the accumulation of organic matter and dead materials. Moreover, its two supply reservoirs serve as troughs for cattle, which in turn causes silting of the dams, increasing the amount of suspended particles in the water, and causing an increase in turbidity before the inlet of water into the ponds.

Guimarães et al., (2009), who evaluated the water quality for tambaqui rearing in similar conditions, observed high variations in turbidity, which varied from 2.20 to 62.1 UNT. Despite such high variations, the authors state that these values are within the acceptable range for tambaqui rearing.

By contrast, Stachiw et al. (2013), who analyzed the water quality of tanks in a fish farm in the municipality of Rolim de Moura, state of Rondônia, obtained low turbidity levels before the inlet of water into the ponds. The authors state that there was an increase in turbidity at the collection points within the ponds, indicating an increase in suspended materials, which is equivalent to the increase in the phytoplanktonic community in the ponds due to fertilization.

\subsection{Ammonia $\left(\mathrm{NH}_{3}\right)$}

Ammonia levels varied between 0.0 and $1.5 \mathrm{mg}$ of $\mathrm{NH}_{3} . \mathrm{L}^{-1}$. No significant differences were observed among October 2015, November 2015, January 2016, February 2016 and May 2016 when compared to the remaining months (Table 1 and Figure 2). In relation to the sampling sites, significant differences were observed among supply reservoir 01, V03 and V04, supply reservoir 02 and V04, 04 and V13 (Table 2 and

Figure 3). Even with the observed variations, these levels agree with those established by Resolution 357/2005/CONAMA (2.18 mg.L-1 for bodies of water used for fishing or aquaculture). 
According to Kubitza (2003), values of non-ionized ammonia above $0.2 \mathrm{mg} . \mathrm{L}^{-1}$ are enough to induce chronic toxicity, leading to a decrease in growth and tolerance of fish to diseases. Ammonia levels between 0.7 and $2.4 \mathrm{mg} . \mathrm{L}^{-1}$ may be lethal to fish even if they are exposed for a short period of time.

Even with ammonia levels considered low and within the standards established in the literature, Abrunhosa (2011) states that levels above $0.2 \mathrm{mg}^{-\mathrm{L}^{-1}}$ are detrimental to fish, causing chronic toxicity depending on exposure time.

Ammonia is a toxic, highly restrictive compound to fish, such that many species cannot endure concentrations above $5 \mathrm{mg} . \mathrm{L}^{-1}$ (CETESB, 2009). Ammonia present in pond water exists in an equilibrium according to temperature and $\mathrm{pH}$, in which ammonia is toxic at low concentrations, and increases according to increases in $\mathrm{pH}$ (QUEIROZ; BOEIRA, 2006).

Moreover, ammonia causes the consumption of dissolved oxygen when biologically oxidated. For this reason, the concentration of ammoniacal nitrogen is an important parameter for the classification of natural waters and is normally used for constituting water quality indexes (CETESB, 2009).

\subsection{Electrical conductivity (EC)}

The electrical conductivity varied from 51.9 to $178.2 \mu \mathrm{S} . \mathrm{cm}^{-1}$, with minimum values in April 2016 in pond 02 , and maximum values in January 2016. In the evaluated months, no significant differences were observed in August 2015, September 2015, October 2015, November 2015, May 2016 and June 2016 when compared to the remaining months (Table 1 and Figure 2). However, in relation to the sampling sites, significant differences among supply reservoirs 01 and V01, V03, V04, V12, V13; supply reservoirs 02, V01, V03, V04, V12, and V13; V02 and V01; V04, V02, V12, and V13 were observed (Table 2 and Figure 3).

The maximum values obtained in this study were above those established by Resolution 357/2005/CONAMA, which establishes that the electrical conductivity must not exceed $100 \mu \mathrm{S} . \mathrm{cm}^{-1}$. These values may also indicate a high degree of organic matter decomposition, and are thus a means to assess nutrient availability in aquatic ecosystems (SIPAÚBA-TAVARES, 1994).

When compared with the values observed in the literature, the EC values of the present study are above those observed by Silva (2014), who carried out limnological analyses in a dam located in the municipality of Presidente Médici, state of Rondônia, with average levels between 48.13 and $55.34 \mu \mathrm{S} . \mathrm{cm}^{-1}$. Such low EC levels may be associated with the fact that the dam is not used for fish rearing.

When compared to studies carried out in fish farming systems, the results agree with those obtained by Costa (2014) who characterized the limnological parameters of a fish farming system with continuous water flow, with EC values ranging from 6.4 to $155.4 \mu \mathrm{S} . \mathrm{cm}^{-1}$. Similar results were also obtained by Paiva (2014) in a fish farming system located in the municipality of Ji-Paraná-RO with values ranging from 66.7 to $157.84 \mu \mathrm{S} . \mathrm{cm}^{-1}$.

Another study, which assessed the limnological parameters in a tambaqui rearing pond, also found high levels of electrical conductivity, with mean levels between 193.3 and $394.33 \mu{\mathrm{S} . \mathrm{cm}^{-1}}^{-1}$ (CAMPECHE et al., 2009). The authors associated this variation to a local rise in temperature, which lead to water evaporation, and, consequently, to an increase in ion concentration.

\subsection{Rainfall data}

Precipitation data showed a maximum of $699.0 \mathrm{~mm}$ in January 2016 and a minimum of $0.0 \mathrm{~mm}$ in November and December 2015 and February and May 2016 . According to Mendonça et al.; (2013), the rainfall scenario in the Amazon Region presents seasonality with high rainfall indexes in certain months. The causality of such events might be associated with meteorological phenomena such as the El Niño, which is characterized by warming of surface waters of the Equatorial Pacific Ocean and by the weakening of the easterly trade winds, thus causing these climate changes (SOUZA et al., 2009).

\section{Final considerations}

The management practice applied by the fish farmer during the study period, together with the dry and rainy seasons and the sequential supply system directly interfered with variations in the evaluated limnological parameters. Most of the parameters, such as ammonia, nitrite, $\mathrm{pH}$, turbidity and dissolved 
oxygen levels were within the limits established by the current legislation during December 2015 in V01 and during October 2015 in V013.

By contrast, the electrical conductivity presented variations above that established by Resolution 357/2005/CONAMA. However, since tambaqui is a very adaptable species, these values did not affect its culture during the nursing phase. Thus, it is recommended that fish farm management fulfill the needs of individual ponds at the end of every rearing cycle, meaning there is a need to substitute sequential supply systems for individual supply systems, which allow for better treatment of any difficulties that might occur in each separate pond.

\section{Acknowledgment}

To Mr. Silveira for allowing the collection of water during the execution of the study, UNIR and $\mathrm{CNPq}$ for scientific initiation fellowships.

\section{Reference}

ABRUNHOSA, F. Técnico em Aquicultura: Piscicultura. Pará: IFPA e UFRN, 2011. Disponível em: http://redeetec.mec.gov.br/images/stories/pdf/eixo_rec_naturais/aquicultura/181012_piscicult.pdf. Acesso em: 7 maio 2018.

AYROZA, D. M. M. R. Características limnológicas em áreas sob influência de piscicultura em tanquesrede no reservatório da UHE Chavantes, rio Paranapanema, SE/S, Brasil. 2012. 130f. Tese (Doutorado), Centro de Aquicultura, Universidade Estadual Paulista, Jaboticabal, 2012.

BALDISSEROTO, B. Fisiologia de peixes aplicada à piscicultura. 3. ed. Santa Maria: ed. da UFMS, 2013. Cap. 08, p. 181-230.

BICUDO, C.E.M.; MENEZES, M. (eds.). Gêneros de algas de águas continentais do Brasil: chave para identificação e descrições. 2. ed. Rima, São Carlos, 2006. 502p.

BOYD, C.E. Manejo do solo e da qualidade da água em viveiro para aquicultura. Campinas: Associação Americana de Soja. (tradução: Eduardo Ono), 1997, 55p.

CAMPECHE, D.F.B.; PEREIRA, L.A.; FIGUEIREDO, R.A.C.R.; PAULINO, R.V.; ALVES, M.A.; NOVA, L.L.M.V.; GUEDES, E.A.C. Limnological parameters and phytoplakton in fshponds with tambaqui, Colossoma macropomum (Cuvier, 1816) in the semi-arid region. Acta Limnologica Brasiliensia v.21, n.3, p.333-341, 2009.

CAVERO, B.A.S.; PEREIRA, M. F.; BORDNHON, A. M.; FONSECA, F. A. L.; ITUASSÚ, D. R.; ROUBACH, R.; ONO, E. A. Tolerância de juvenis de pirarucu ao aumento da concentração de amônia em ambiente confinado. Pesquisa Agropecuária Brasileira. v.39, n. 5, p. 513-516, 2004.

CETESB. Qualidade das águas interiores no Estado de São Paulo. Significado ambiental e sanitário das Variáveis de qualidade das águas e dos Sedimentos e metodologias analíticas e de amostragem. Relatório Técnico CETESB. 2009. p. 1-44. Disponível em: http://cetesb.sp.gov.br/aguas-interiores/wpcontent/uploads/sites/32/2013/11/variaveis.pdf. Acesso em: 20 de junho de 2017.

CECCARELLI, P. S.; SENHORINI, J. A.; VOLPATO, G. Dicas em Piscicultura: perguntas e respostas. Santana ed., Botucatu - SP, p. 247, 2000.

CONAMA - Conselho Nacional do Meio Ambiente. Resolução CONAMA no 357 de 17 de Março de 2005. Dispõe sobre a classificação dos corpos d'água e diretrizes ambientais para o seu enquadramento, bem como estabelece as condições e padrões de lançamento de efluentes. Diário Oficial da República Federativa do Brasil, Brasília, DF, 18 de março de 2005. 
COSTA, R.L. Análise dos parâmetros liminológicos e da comunidade fitoplanctônica dos viveiros da Piscicultura Santa Helena, Alvorada D' Oeste, RO. 2014. 84f. Monografia (Bacharelado em Engenharia de Pesca), Departamento de Engenharia de Pesca, Fundação Universidade Federal de Rondônia, Presidente Médici, 2014.

ESTEVES, F.A.; FURTADO, A.LS. Oxigênio Dissolvido. In: Fundamentos de Limnologia. ESTEVES, F. A. (Coordenador). $3^{\text {a }}$ Ed. Editora Interciência, Rio de Janeiro - RJ. p. 167-191. 2011.

FAO - FOOD AND AGRICULTURE ORGANIZATION. The state of world fisheries and aquaculture: opportunities and challenges. Rome: FAO, 2016. 243 p.

GUERREIRO, L.R.J. Custo de Produção, Análise Econômica e Gerencial em Unidade de Produção de Alevinos de Peixes Reofílicos: Estudo de caso em Rondônia. 2012. 150 f. Dissertação (Mestrado em Zootecnia - Área de concentração Produção Animal) - Universidade Federal do Rio Grande do Sul. 2012.

GUIMARÃES, C.E.P.; CARNEIRO, L.B.; HERMÓGENES, L.S.; PINHEIRO, D.M.; MIRANDA, E.C.; FÉLIX, M.C. Avaliação da qualidade de água de viveiros utilizados para cultivo de tambaqui no CERAQUA/CODEVASF. In: Reunião anual da Sociedade Brasileira de Química, 33르 Anais... 2009. Disponível em: http://sec.sbq.org.br/cdrom/34ra/resumos/T1606-2.pdf Acesso em: 02 mar. de 2018.

HURTADO, F.B.; FIGUEIREDO, F.M.; DA COSTA, R. L.; BOMFIM, S. C.; DE QUEIROZ, C.B.; PONTES, W.P. Parâmetros limnológicos em viveiros de piscicultura semi-intensiva de tambaqui com abastecimento em disposição sequencial. Revista em Agronegócio e Meio Ambiente, v. 11, n. 1, p. 9-30, 2018.

IBGE - Instituto Brasileiro de Geografia e Estatística. Produção da Pecuária Municipal. Brasil: IBGE, 2016. Disponível em: https://biblioteca.ibge.gov.br/index.php/biblioteca-catalogo?view=detalhes\&id=784. Acesso em: 9 maio 2018.

KUBITZA, F. Qualidade da água no cultivo de peixes e camarões. Jundiaí, 229p. 2003.

MAR, C.C.; OLIVEIRA, L.M.; COSTA, A.D. Perfil Socioeconômico dos Piscicultores de Capitão Poço. In: Seminário Anual de Iniciação Científica da UFRA. Manaus - AM. 2012.

MDIC - Ministério do Desenvolvimento, Indústria e Comércio Exterior. Arranjo produtivo local da piscicultura de Pimenta Bueno - RO. Porto Velho. 2007. Disponível em: http://www.desenvolvimento.gov.br/arquivos/dwnl_1248265904.pdf. Acesso em: mar. 2018.

MENDONÇA, A.P.; CARVALHO, F.R.; SOUSA, F.F.G.; LEITE, K.G.; FEND, L.C.P. Variações mensais e interanuais de precipitação pluviométrica no município de Ouro Preto D'Oeste-RO, Amazônia Ocidental. Revista de Desenvolvimento e Inovação, v. 1, n. 1, p.66-72, 2013.

MORO, G. V.; RODRIGUES, A. P. O.; TORATI, L. S.; BARROSO, R. M.; LUNDSTEDT, L.M. Anatomia e fisiologia de peixes de água doce. In: RODRIGUES, A. P. O. et al. Editores técnicos. Piscicultura de água doce: multiplicando conhecimentos. Brasília - DF: Embrapa, p 71-95. 2013.

NAKATANI, K.; AGOSTINHO, A.A.; BAUMGARTNER, G.; BIALETZKI; SANCHES, P.V.; MAKRAKIS, M.C.; PAVANELLI, C.S. Ovos e larvas de peixes de água doce - Desenvolvimento e Manual de Identificação. Editora da UEM. Maringá-PR. 2001.

PAGGI, L.C. Avaliação limnológica em um sistema de piscicultura na região de Paranaíta (MT, Brasil). Jaboticabal: UNESP, 2006. Dissertação (Mestrado em Aquicultura), centro de aquicultura da UNESP, Universidade Estadual Paulista, 2006. 
PAIVA, M.C. Análise da qualidade da água de um sistema de piscicultura: estudo de caso no município de Ji-Paraná / RO - Brasil. 2014. 89f. Monografia (Bacharelado em Engenharia Ambiental), Departamento de Engenharia Ambiental, Fundação Universidade Federal de Rondônia, Ji-Paraná, 2014.

PARRON, L.M.; MUNIZ, D.H.F.; PEREIRA, C.M. Manual de procedimentos de amostragem e análise físico química de água. Colombo: Embrapa florestas. 2011.69p.

PEREIRA, C.A.; RESENDE, J.J.; BARATTO, A.J.; BORGES, E.S.M.; CARVALHO, G.W. A. Avaliação dos parâmetros limnológicos da lagoa e de um viveiro escavado da piscicultura no IF sudeste MG / Campus Barbacena: avaliação visando boas práticas de manejo. In: Simpósio de Pesquisa e Inovação III/ISeminário de Iniciação Científica, II. 2012, Barbacena, Anais... Barbacena, IF Sudeste de Minas Gerais, v. 1, n. 1, 2012. p. 71-75.

PIANA, P.A.; BAUMGARTNER, G.; GOMES, L.C. Influência da temperatura sobre o desenvolvimento de juvenis de piapara (Leporinus cf. obtusidens). Acta Scientiarum: Biological Sciences. v. 25, p:87-94. 2003.

QUEIROZ, J.F.; BOEIRA, R.C. Calagem e Controle da Acidez dos Viveiros de Aquicultura. Jaguariúna: Embrapa Meio Ambiente, 2006. 8p. (Embrapa Meio Ambiente. Circular Técnica, 14).

QUEIROZ. J.F.; SIVEIRA, M.P. Recomendações Práticas para Melhorar a Qualidade da Água e dos efluentes dos viveiros de aquicultura. Jaguariúna: Embrapa Meio Ambiente, 2006. 14p. (Embrapa Meio ambiente Circular Técnica, 12).

SCHULTER, E.P.; VIEIRA FILHO, J.E.R. Evolução da piscicultura no Brasil: Diagnóstico e desenvolvimento da cadeia produtiva de tilápia. Texto para Discussão, 2017.

SILVA, S.M. Avaliação Limnológica da represa e efluente da base de piscicultura Carlos Eduardo Matiazze: Presidente Médici-RO: UNIR, 2014. 57f. Monografia (Bacharelado em Engenharia de Pesca), Departamento de Engenharia de Pesca, Fundação Universidade Federal de Rondônia, 2014.

SILVA, C.A.; CARNEIRO, P. Qualidade da água na engorda de tambaqui em viveiros sem renovação da água. Ed. Gomes, Aracaju - SE, 2007.

SILVA, C.A. da; FUJIMOTO, R.Y. Crescimento de tambaqui em resposta a densidade de estocagem em tanques-rede. Acta Amazonica, v.45, n. 3, p.323-332, 2015.

SILVA, F.N. L.; DE MEDEIROS, L.R., DA COSTA, M.S.M.; MACEDO, A.R.G.; BRANDÃO, L.V.; DE SOUZA, R.A.L. Qualidade da água proveniente de poço artesiano em viveiro de piscicultura. PUBVET, v. 11, p. 646-743, 2017.

SIPAÚBA-TAVARES, L.H. Limnologia aplicada à aquicultura. FUNEP, São Paulo - SP, 72 p, 1994.

SOUZA, S.M. Avaliação limnológica em reservatórios: estudo de caso do cultivo de tilápias em raceways, Paulo Afonso - BA. 2007. 49f. Dissertação (Mestrado em Recursos Pesqueiros e Aquicultura). Universidade Federal Rural de Pernambuco. 2007. 